\title{
Full N,N-Methylation of 4,4'-Methylenedianiline with Dimethyl Carbonate: A Feasible Access to 4,4'-Methylene bis(N,N-Dimethylaniline)
}

\author{
Zegang Qiu $\mathbb{D}^{1},{ }^{1}$ Kunjie Wang, ${ }^{2}$ Zhiqin Li $\mathbb{D},{ }^{1}$ Tao Li, ${ }^{1}$ Jinhao Bai, ${ }^{1}$ Chanjuan Yin, \\ Xiushen Ye, ${ }^{3}$ and Haining Liu $^{3}$ \\ ${ }^{1}$ College of Chemistry and Chemical Engineering, Xi'an Shiyou University, Xi'an 710065, China \\ ${ }^{2}$ Xian Aerospace Composites Research Institute, Xian 710025, China \\ ${ }^{3}$ The Qinghai Institute of Salt Lakes, The Chinese Academy of Sciences, Xining 810008, China \\ Correspondence should be addressed to Zhiqin Li; lizhiqin@xsyu.edu.cn
}

Received 25 December 2017; Accepted 14 February 2018; Published 18 April 2018

Academic Editor: Bartolo Gabriele

Copyright (c) 2018 Zegang Qiu et al. This is an open access article distributed under the Creative Commons Attribution License, which permits unrestricted use, distribution, and reproduction in any medium, provided the original work is properly cited.

The full N,N-methylation of $4,4^{\prime}$-methylenedianiline (MDA) with dimethyl carbonate (DMC) was investigated. The yield of the major product $4,4^{\prime}$-methylene bis(N,N-dimethylaniline) (MBDMA) reached as high as $97 \%$ over NaY catalyst at $190^{\circ} \mathrm{C}$ for $6 \mathrm{~h}$. The catalyst could be used for two more times with acceptable MBDMA yields higher than $90 \%$. The main by-products were identified as three $\mathrm{N}$-methylated derivatives. Surprisingly, the formation of the N-methoxycarbonylation product was extremely restrained, which could be produced in high yields of $98 \%$ on zinc acetate catalyst. Furthermore, the reaction pathway to the major product MBDMA was proposed. Finally, a feasible synthetic route of $4,4^{\prime}$-methylene bis(N,N-dimethylaniline) (MBDMA) was established, featuring a high yield, mild reaction conditions, and simple operations.

\section{Introduction}

The reaction of $4,4^{\prime}$-methylenedianiline (MDA) with dimethyl carbonate (DMC) produced $4,4^{\prime}$-methylenedimethyldiphenylcarbamate (MDC) in high yield of $98 \%$, under the catalysis of the zinc acetate, as described in literatures [1, 2 ] and our previous work [3]. Furthermore, the results of our research demonstrated that the three main by-products formed during the $\mathrm{N}$-methoxycarbonylation reaction were all N-methylated derivatives [3]. Such results revealed that the $\mathrm{N}$-methoxycarbonylation and the $\mathrm{N}$-methylation were two competitive processes in the reaction of MDA with DMC. So far, almost all of the research focused on the $\mathrm{N}$-methoxycarbonylation process between MDA and DMC, which was always treated as the main reaction; accordingly, the details of N-methylation process were still left unknown. Thus, we moved our focus to the $\mathrm{N}$-methylation reaction of MDA with DMC and tried to make it to be the predominant reaction. Two possible $\mathrm{N}$-methylation reaction pathways were listed in Scheme 1. Our purpose was to produce $4,4^{\prime}$-methylene bis(N,N-dimethylaniline) (MBDMA) or 4, $4^{\prime}$ methylene-bis-(N-methylaniline) (MBMA) or the both in high yield.

Aforementioned MBDMA has found many applications in many fields. For example, it can be used as an intermediate in dye manufacture and as an analytical reagent in the determination of lead, iodide, bromide, and so forth [4-6]. In addition, it can also form charge-transfer complex with phenazine and its derivatives [7-9]. Commercially, MBDMA was synthesized by condensation of N,N-dimethyl aniline with formaldehyde over homogeneous acid catalysts [10]. However, this route suffered from some problems such as process complexity, equipment corrosion, and environmental pollution caused by the waste produced. Recently, ionic liquid was used in the reaction of $\mathrm{N}, \mathrm{N}$-dimethylaniline with tetrachloromethane to produce MBDMA in the highest yield 


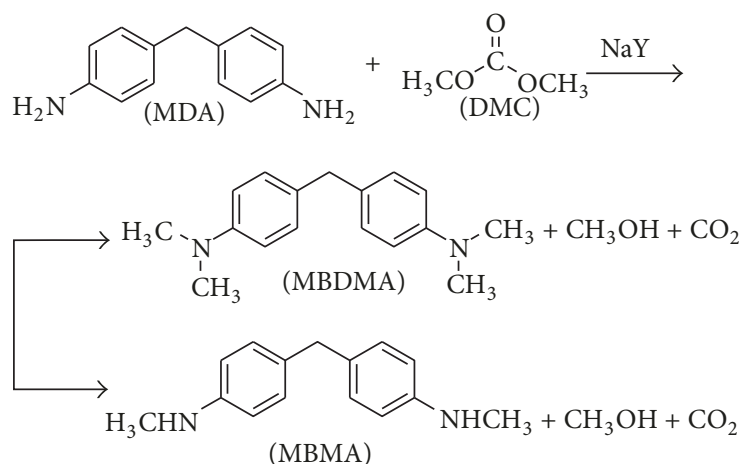

(MBMA)

SCHEme 1: Two possible N-methylation reaction pathways of MDA with DMC.

of $61 \%$ [11]. Also, the structure and vibrational spectra of MBDMA were investigated by the DFT-B3LYP and ab initio MP2 calculations [12].

The reactant MDA has been produced on industrial scale, while DMC is a versatile methylating or methoxycarbonylating reagent for the green synthesis of many organics [13]. The N-methylation of primary amines with DMC has been extensively investigated [14-20]; in particular, a general protocol for the reductive $\mathrm{N}$-methylation of primary and secondary amines using dimethyl carbonate and molecular hydrogen was developed recently [21]. It is worth noting that the mono-N-methylation of primary aromatic amines with dimethyl carbonate could occur in high selectivity $[15,16]$. However, the N,N-methylation or mono-N-methylation of diamines with DMC has not been described. Therefore, works should be done to figure out how and to what extent the N,Nmethylation or mono-N-methylation reaction of diamines with DMC occurs. Furthermore, a successful synthesis of MBDMA from MDA and DMC should not only set an example of the N,N-methylation reaction of diamine with DMC but also provide a new alternative route to MBDMA.

In this paper, $4,4^{\prime}$-methylene bis(N,N-dimethylaniline) (MBDMA) was obtained by the $\mathrm{N}, \mathrm{N}$-methylation reaction of $4,4^{\prime}$-methylenedianiline (MDA) with dimethyl carbonate (DMC) in high yield, under the catalysis of $\mathrm{NaY}$ zeolite. Also, the main by-products formed during the N,N-methylation process were identified. The reusability of $\mathrm{NaY}$ catalyst was checked. Furthermore, the formation mechanism of the Nmethylated compounds was discussed. However, the effort to produce MBMA failed, and the details will be provided later in this paper.

\section{Experimental}

2.1. Chemicals. 4,4-Methylenedianiline (MDA) was commercially available and used without further treatment. DMC of analytically pure grade was purified by distillation before use. The $\mathrm{H} \beta$, ZSM-5, NaY, NaX, and MCM-41 were commercial products from the Catalyst Plant of Nankai, and they were calcined at $500^{\circ} \mathrm{C}$ for $2 \mathrm{~h}$ before use.

2.2. Instrumentation. High performance liquid chromatography (HPLC) analyses were performed on a Shimadzu
LC (Japan) equipped with a SPD-10Avp detector. A Shimpack Vp-ODS column $(150 \times 4.6 \mathrm{~mm})$ and a mobile phase $\mathrm{CH}_{3} \mathrm{OH} / \mathrm{H}_{2} \mathrm{O} 60 / 40$ (volume) were used. ${ }^{1} \mathrm{H}$ NMR spectra were recorded on a Bruker drx-300 instrument (Germany). Chemical shifts were reported in ppm $(\delta$-scale) relative to internal standard TMS; $\mathrm{CDCl}_{3}$ was used as a solvent. IR spectra $\left(\mathrm{cm}^{-1}\right)$ were collected with a Thermo Nicolet 380 FT-IR (USA). X-ray diffraction (XRD) measurements were performed on a Bruker D8 Advance X-Ray Diffractometer (Germany) with a $\mathrm{Cu} \mathrm{K} \alpha$ radiation source. Elemental analysis was performed with an Elementar Vario EL (Germany). HPLC coupled with mass spectrometry (HPLC/MS) was run on a Waters HPLC/MS system, equipped with a Waters alliance 2695 HPLC pump, a Waters 2996 photodiode array detector, and a Waters micromass ZQ-4000 mass spectrometer. A Waters symmetry C18 column $(150 \mathrm{~mm} \times 211 \mathrm{~mm}$, $3 \mu \mathrm{m}$ ) and a mobile phase $\mathrm{CH}_{3} \mathrm{OH} / \mathrm{H}_{2} \mathrm{O} 65 / 45$ (volume) were used. The flow rate of the mobile phase was $3 \mathrm{~mL} / \mathrm{min}$. The ion source temperature was $130^{\circ} \mathrm{C}$ and the cone temperature was $20^{\circ} \mathrm{C}$. $\mathrm{N}_{2}$ adsorption-desorption isotherms were recorded on a Tristar-3000 Micromeritics volumetric apparatus. The special surface area was calculated according to the BET isothermal equation. The temperature-programmed desorption of ammonia $\left(\mathrm{NH}_{3}\right.$-TPD) was carried out. About $100 \mathrm{mg}$ sample (20-40 mesh) was pretreated at $500^{\circ} \mathrm{C}$ for $2 \mathrm{~h}$ in a quartz tube in nitrogen stream. Then, it was cooled to $100^{\circ} \mathrm{C}$ and adsorbed ammonium for $10 \mathrm{~min}$. The desorption of ammonium was conducted at a heating rate of $10^{\circ} \mathrm{C} / \mathrm{min}$ in nitrogen flow $(30 \mathrm{~mL} / \mathrm{min})$. The desorbed ammonium was monitored by a thermal conductivity detector (TCD).

2.3. Reaction. All the reactions were conducted in a $100 \mathrm{~mL}$ stainless autoclave with a magnetic stirrer. MDA, DMC, and catalysts were charged into the reactor. The air in the autoclave was fully replaced with nitrogen to guarantee that the reaction was carried out under the inert atmosphere. The mixture was then stirred constantly and heated to a selected temperature for certain hours. When the reaction was completed, the autoclave was cooled down to room temperature. The solid-liquid mixture obtained was separated into two parts by filtration: a white powder (zeolite catalyst) and a clear light yellow liquid. The liquid was then analyzed with Shimadzu HPLC using naphthalene as an internal standard. 
TABLE 1: Formation of MBDMA over various catalysts.

\begin{tabular}{lccc}
\hline Catalysts & $\begin{array}{c}\text { MDA } \\
\text { Conversion } \\
/ \%\end{array}$ & $\begin{array}{c}\text { MBDMA } \\
\text { Sectivity }\end{array}$ & $\begin{array}{c}\text { Yield } \\
/ \%\end{array}$ \\
\hline None & 61.2 & None & None \\
$\mathrm{H} \beta$ & 61.8 & None & None \\
ZSM-5 & 62.2 & None & None \\
NaX & 100 & 28.7 & 28.7 \\
NaY & 100 & 91.1 & 91.1 \\
MCM-41 & 86.4 & None & None \\
\hline
\end{tabular}

Reaction Conditions. DMC $27.0 \mathrm{~g}(0.30 \mathrm{~mol}), \mathrm{MDA} 1.98 \mathrm{~g}(0.01 \mathrm{~mol})$, catalyst $1.98 \mathrm{~g}$, reaction time $6 \mathrm{~h}$, and reaction temperature $180^{\circ} \mathrm{C}$.

The conversion of MDA and the yield of MBDMA were calculated by HPLC.

2.4. Characterization of MBDMA. A MBDMA yield of $97 \%$ $(2.47 \mathrm{~g}, 0.0097 \mathrm{~mol})$ and a MDA conversion of $100 \%$ were attained, when a mixture of MDA (1.98 g, $0.01 \mathrm{~mol}), \mathrm{DMC}$ $(27.0 \mathrm{~g}, 0.30 \mathrm{~mol})$, and $\mathrm{NaY}(1.98 \mathrm{~g})$ was stirred at $190^{\circ} \mathrm{C}$ for $6 \mathrm{~h}$. In this case, the liquid was a mixture consisting of MBDMA, DMC, and a tiny amount of by-products. After distilling off DMC from the mixture, a light yellow solid was obtained. The solid was further purified by recrystallization from alcohol, and a crystalline compound was obtained which was identified as MBDMA.

Characterization data of MBDMA were listed as follows. 4,4' ${ }^{\prime}$-Methylene bis(N,N-dimethylaniline) (MBDMA): IR (KBr) $v / \mathrm{cm}^{-1} 3448,2886,2804,1613,1564,1521,1480,1444$, $1355,1342,1309,1231,1189,1168,1124,1071,949,829,795,568$, $508 ;{ }^{1} \mathrm{H}$ NMR $\left(300 \mathrm{MHz}, \mathrm{CDCl}_{3}\right) \delta 2.88\left(\mathrm{~s}, 12 \mathrm{H}, \mathrm{CH}_{3}\right), 3.79$ (s, $\left.2 \mathrm{H}, \mathrm{CH}_{2}\right), 6.66-6.68(\mathrm{~m}, 4 \mathrm{H}, \mathrm{Ar}-\mathrm{H}), 7.03-7.06(\mathrm{~m}, 4 \mathrm{H}$, Ar-H); MS, $m / z$, observed: $255.3[\mathrm{M}]^{+}+1, \mathrm{C}_{17} \mathrm{H}_{22} \mathrm{~N}_{2}[\mathrm{M}]^{+}$ +1 requires: 255.4. For $\mathrm{C}_{17} \mathrm{H}_{22} \mathrm{~N}_{2}$ (254.4); found: $79.94 \% \mathrm{C}$, $8.63 \% \mathrm{H}, 10.73 \% \mathrm{~N}$. requires: $80.27 \% \mathrm{C}, 8.72 \% \mathrm{H}, 11.01 \% \mathrm{~N}$.

2.5. The Analysis of By-Products. In order to obtain a suitable amount of by-products for analyzing, a mixture of MDA $(1.98 \mathrm{~g}, 0.01 \mathrm{~mol}), \mathrm{DMC}(27.0 \mathrm{~g}, 0.30 \mathrm{~mol})$, and $\mathrm{NaY}(1.98 \mathrm{~g})$ was stirred at $150^{\circ} \mathrm{C}$ for $6 \mathrm{~h}$, attaining a MBDMA yield of $11.4 \%$ and a MDA conversion of $100 \%$. The liquid obtained after filtering off the catalyst was then analyzed with HPLC/MS.

\section{Results and Discussion}

3.1. Catalyst Function. As the zeolites exhibited effective catalytic activity to the methylation reaction of some aromatic amines [16, 17, 22-24], several different zeolites such as $\mathrm{H} \beta$, H-ZSM-5, NaX, NaY, and MCM-41 were chosen to catalyze the reaction of MDA with DMC in order to obtain MBDMA, as shown in Table 1. Disparate catalytic performances were observed. $\mathrm{H} \beta$ and H-ZSM-5 exhibited almost no activity; MCM-41 facilitated the conversion of MDA but showed no selectivity to the MBDMA; NaX had better activity to the conversion of MDA but poor selectivity to MBDMA; $\mathrm{NaY}$

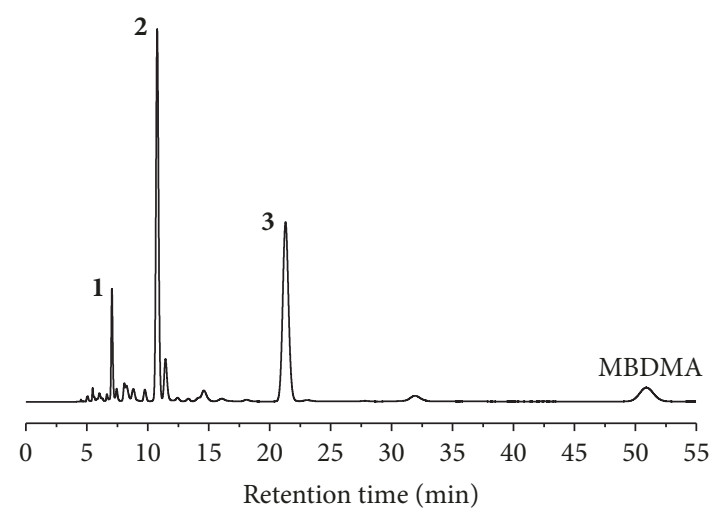

FIgURE 1: The liquid chromatogram of the filtrate obtained after the reaction of MDA with DMC.

had the best activity and selectivity among the chosen zeolites. Under the catalysis of NaY, MDA could be completely transformed and MBDMA selectivity reached a high value of 91.1\%. Such results revealed that the formation of MBDMA depended on the properties of the catalysts. The properties such as the pores and structures of $\mathrm{H} \beta, \mathrm{ZSM}-5, \mathrm{NaX}, \mathrm{NaY}$, and MCM- 41 are apparently different. $\mathrm{H} \beta$ and $\mathrm{H}-\mathrm{ZSM}-5$ have micropores but have no cages, and $\mathrm{NaX}$ and $\mathrm{NaY}$ possessed micropores as well as octahedral zeolite cages, while MCM-41 possesses mesopores and orderly lined hexagonal channels. Therefore, it could be broadly inferred that the formation of MBDMA is associated with the pore sizes and structures. As $\mathrm{NaY}$ exhibited outstanding catalytic performance, further investigation was made to improve the yield of MBDMA and to clarify the reaction mechanism.

3.2. The By-Products Formed over NaY. To help to comprehend the formation mechanism of the main product, the byproducts in the reaction of MDA with DMC over NaY were analyzed by HPLC/MS, obtaining the liquid chromatogram (Figure 1) and the MS spectra (Figure 2). Three by-products including 1, 2, and 3 were detected as shown in Figure 1. The molecular weights of compounds $\mathbf{1}, \mathbf{2}$, and $\mathbf{3}$ could be obtained by analyzing the MS spectra, which were listed in Table 2. The possible configurations of $\mathbf{1}, \mathbf{2}$, and $\mathbf{3}$ could be sketched (shown in Table 2), based on the molecular weights and the known compounds (MDA and DMC) added in the reaction system. As we can see, compounds $\mathbf{1}$ and $\mathbf{3}$ only corresponded to $\mathbf{1 a}$ and $\mathbf{3 a}$, respectively. Thus, the structures of compounds $\mathbf{1}$ and $\mathbf{3}$ were clearly identified (shown in Table 2), by combining the analyses of the fragment ions in the corresponding MS spectra. However, the molecular weights of compound 2 corresponded to two possible configurations $\mathbf{2 a}$ and $\mathbf{2 b}$. Therefore, meticulous analyses to MS spectra of 2 in Figure 2(b) were done to further identify its structure. Fortunately, a key ion 4 at $\mathrm{m} / z 106$ (Figure 2(b)) was found, and it could be generated by $\mathbf{2 a}$ but not by $\mathbf{2 b}$. Furthermore, most of the fragment ions in Figure 2(b) could be ascribed to compound $\mathbf{2 a}$. Thus, compound $\mathbf{2}$ was confirmed to be $\mathbf{2 a}$. So far, the by-products $\mathbf{1}, \mathbf{2}$, and $\mathbf{3}$ were finally identified as 


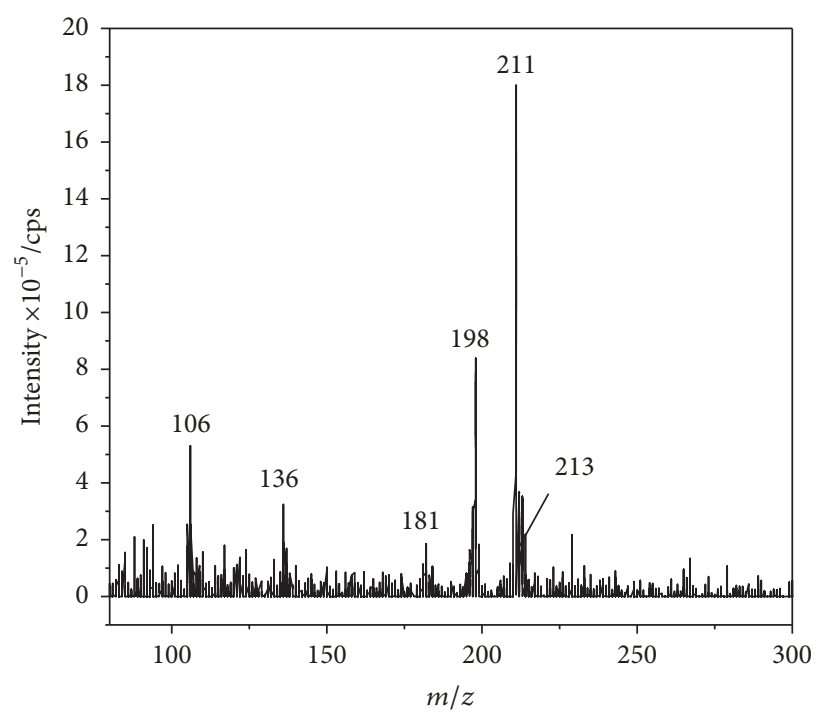

(a)

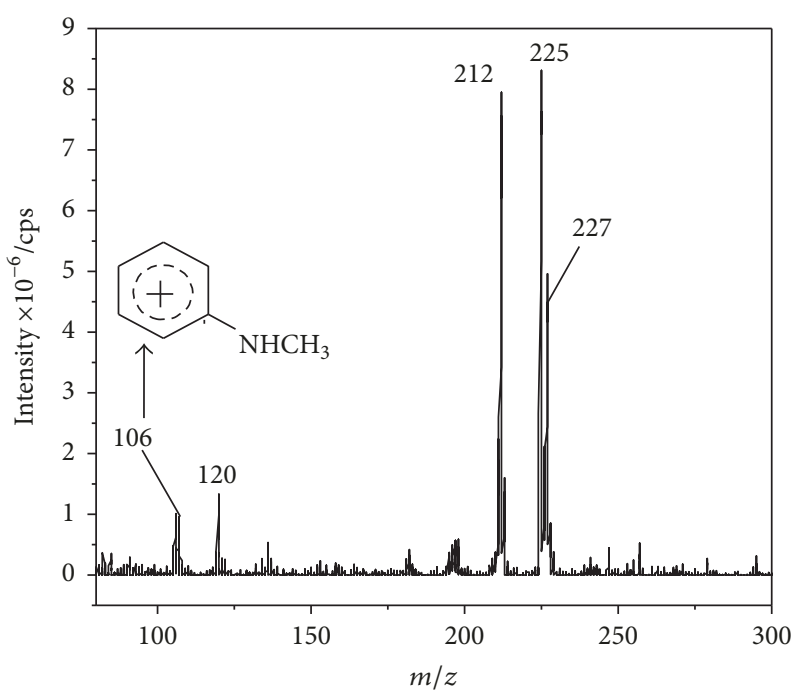

(b)

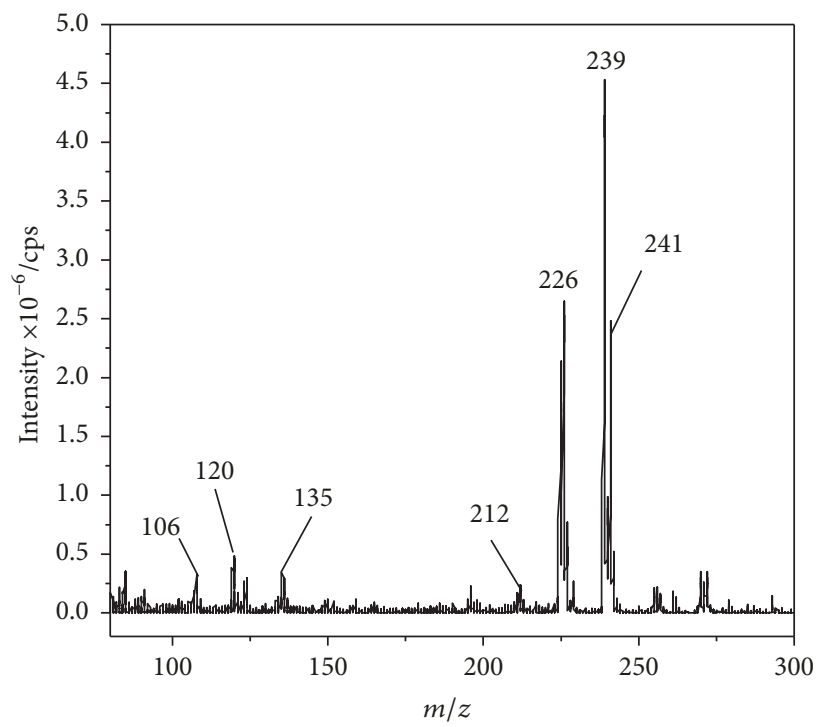

(c)

Figure 2: MS spectra of the by-products 1, 2, and 3. Subfigures (a), (b), and (c) present the MS spectra of the pseudomolecular ions of compounds $\mathbf{1}, \mathbf{2}$, and $\mathbf{3}$, respectively. $\mathbf{4}$ stands for the ion with an $\mathrm{m} / \mathrm{z}$ value of 106 .

three $\mathrm{N}$-methylated derivatives with different degrees of $\mathrm{N}$ methylation, as shown in Table 2.

3.3. The Optimum Reaction Conditions for the Formation of MBDMA. The effects of the reaction conditions including the reaction temperature, the reaction time, the $\mathrm{NaY}$ amount, and the DMC/MDA molar ratio on the formation of MBDMA over NaY zeolites were investigated, and the results were displayed in Table 3 and Figures 3 and 4 .

The NaY amount was expressed by NaY/DMC weight ratio. It was found that a ratio higher than 0.07 was needed to warrant the high MBDMA yields (Table 3). In this reaction, DMC was taken in excess to fully utilize MDA and facilitate the product formation. DMC served as both the reagent and the solvent. The optimum DMC/MDA molar ratio was
30 (Table 3). The excess DMC distilling from the reaction mixture could be recycled.

As shown in Table 3, when the reaction temperature was $120^{\circ} \mathrm{C}$, the conversion of MDA was at a low value of $61.2 \%$, while the main product MBDMA was not detected. In this case, the converted MDA mainly produced by-products 1 and 2 (Figure 3). As the reaction temperature was increased, the conversion of MDA and the yield of MBDMA were accordingly increased. When the reaction temperature rose to $160^{\circ} \mathrm{C}$, the conversion of MDA attained $100 \%$; however, the selectivity of MBDMA was only $39.2 \%$. Such poor MBDMA selectivity could be mostly attributed to the formation of the by-products 2 and 3 (Figure 3). When the reaction temperature was going on increased to $190^{\circ} \mathrm{C}$, the selectivity of MBDMA reached to the highest value of $97.4 \%$; on the 
TABLE 2: The molecular weights and structures of by-products $\mathbf{1}, \mathbf{2}$, and 3 .

Compound $\begin{gathered}\text { Molecular } \\ \text { weights }\end{gathered}$

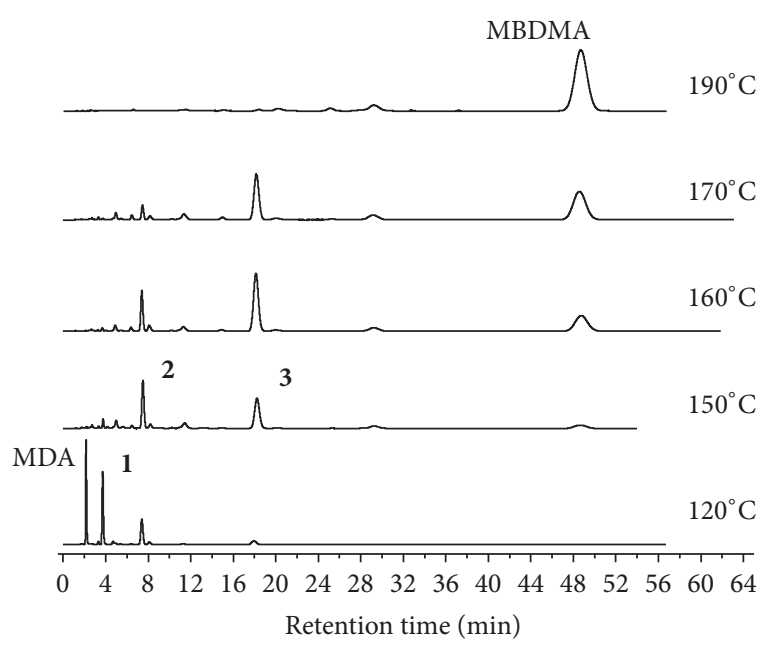

FIGURE 3: Liquid chromatograms obtained after the reaction between MDA and DMC under different temperatures. Reaction conditions: DMC $27.0 \mathrm{~g}(0.30 \mathrm{~mol}), \mathrm{MDA} 1.98 \mathrm{~g}(0.01 \mathrm{~mol}), \mathrm{NaY}$ $1.98 \mathrm{~g}$, and reaction time $6 \mathrm{~h} . m(\mathrm{NaY}) / m(\mathrm{DMC})(\mathrm{g} / \mathrm{g})=0.07 / 1$.

contrary, the unwanted by-products were minimized to very small amount. Further increasing the reaction temperature to $200^{\circ} \mathrm{C}$ could not improve the selectivity of MBDMA anymore. Thus, the optimum reaction temperature should be controlled around $190^{\circ} \mathrm{C}$. Furthermore, the yields of by-products 1,2 , and 3 sequentially increased and then dropped one by one; such trend could effectively prove that the MBDMA was formed via the gradually $\mathrm{N}$-methylated reaction.

As we can see from Table 3 , when the reaction was carried out for $0.5 \mathrm{~h}$, MDA had been fully converted; however, the MBDMA selectivity was at a low value of $26.6 \%$; this was mainly because of the formation of by-products 2 and 3 (Figure 4). As the reaction time prolonged to $2 \mathrm{~h}$, the

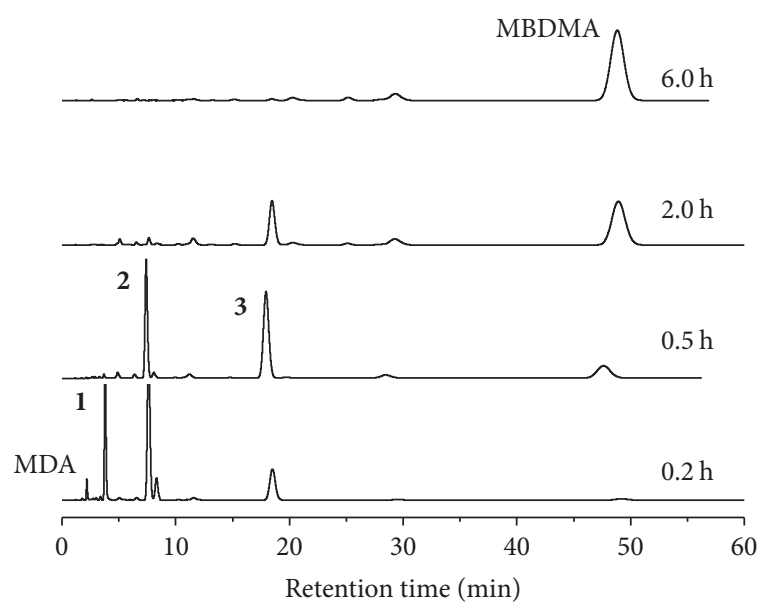

FIGURE 4: Liquid chromatograms obtained after the reaction between MDA and DMC under different reaction times. Reaction conditions: DMC $27.0 \mathrm{~g}(0.30 \mathrm{~mol}), \mathrm{MDA} 1.98 \mathrm{~g}(0.01 \mathrm{~mol}), \mathrm{NaY}$ $1.98 \mathrm{~g}$, and reaction temperature $190^{\circ} \mathrm{C} . m(\mathrm{NaY}) / m(\mathrm{DMC})(\mathrm{g} / \mathrm{g})=$ $0.07 / 1$.

MBDMA selectivity rose to $65.1 \%$; in this case, the predominant by-product was 3 (Figure 4 ). As the reaction time reached to $6 \mathrm{~h}$, the highest MBDMA selectivity of $97.4 \%$ was achieved with a tiny amount of by-products formed. A longer reaction time could not improve the MBDMA selectivity anymore. So the optimum reaction time should be controlled around $6 \mathrm{~h}$. The changing trend of the yields of by-products $\mathbf{1}, \mathbf{2}$, and $\mathbf{3}$ over the reaction time was very similar to that over the reaction temperature. This further proved that the MBDMA was formed following the gradually N-methylated reaction route.

3.4. The Reusability of the Catalyst NaY. In order to check the reusability, the used $\mathrm{NaY}$ catalyst was filtered out, dried 
TABLE 3: The effect of the reaction conditions on the formation of MBDMA over NaY zeolites.

\begin{tabular}{|c|c|c|c|c|c|c|c|}
\hline \multirow[b]{2}{*}{ Run } & \multirow{2}{*}{$\begin{array}{c}\text { Reaction } \\
\text { temperature } /{ }^{\circ} \mathrm{C}\end{array}$} & \multirow{2}{*}{$\begin{array}{l}\text { Reaction } \\
\text { time/h }\end{array}$} & \multirow{2}{*}{$\begin{array}{c}m(\mathrm{NaY}) / m(\mathrm{DMC}) \\
\mathrm{g} / \mathrm{g}\end{array}$} & \multirow{2}{*}{$\begin{array}{c}\mathrm{n}(\mathrm{DMC}) / \mathrm{n}(\mathrm{MDA}) \\
\mathrm{mol} / \mathrm{mol}\end{array}$} & \multirow{2}{*}{$\begin{array}{c}\text { MDA } \\
\text { conversion } \\
/ \%\end{array}$} & \multicolumn{2}{|c|}{ MBDMA } \\
\hline & & & & & & $\begin{array}{l}\text { Yield } \\
/ \%\end{array}$ & $\begin{array}{c}\text { Selectivity } \\
/ \%\end{array}$ \\
\hline 1 & 120 & 6 & 0.07 & 30 & 61.2 & 0.0 & 0.0 \\
\hline 2 & 150 & 6 & 0.07 & 30 & 96.5 & 22.7 & 23.5 \\
\hline 3 & 160 & 6 & 0.07 & 30 & 100 & 39.2 & 39.2 \\
\hline 4 & 170 & 6 & 0.07 & 30 & 100 & 56.5 & 56.5 \\
\hline 5 & 180 & 6 & 0.07 & 30 & 100 & 91.1 & 91.1 \\
\hline 6 & 190 & 6 & 0.07 & 30 & 100 & 97.4 & 97.4 \\
\hline 7 & 200 & 6 & 0.07 & 30 & 100 & 97.5 & 97.5 \\
\hline 8 & 190 & 0.2 & 0.07 & 30 & 96.1 & 13.2 & 13.7 \\
\hline 9 & 190 & 0.5 & 0.07 & 30 & 100 & 26.6 & 26.6 \\
\hline 10 & 190 & 2 & 0.07 & 30 & 100 & 65.1 & 65.1 \\
\hline 11 & 190 & 4 & 0.07 & 30 & 100 & 94.2 & 94.2 \\
\hline 12 & 190 & 6 & 0.07 & 30 & 100 & 97.4 & 97.4 \\
\hline 13 & 190 & 7 & 0.07 & 30 & 100 & 97.4 & 97.4 \\
\hline 14 & 190 & 6 & 0.01 & 30 & 100 & 67.1 & 67.1 \\
\hline 15 & 190 & 6 & 0.03 & 30 & 100 & 96.2 & 96.2 \\
\hline 16 & 190 & 6 & 0.05 & 30 & 100 & 96.8 & 96.8 \\
\hline 17 & 190 & 6 & 0.07 & 30 & 100 & 97.4 & 97.4 \\
\hline 18 & 190 & 6 & 0.09 & 30 & 100 & 97.5 & 97.5 \\
\hline 19 & 190 & 6 & 0.07 & 15 & 100 & 87.6 & 87.6 \\
\hline 20 & 190 & 6 & 0.07 & 20 & 100 & 96.2 & 96.2 \\
\hline 21 & 190 & 6 & 0.07 & 25 & 100 & 96.5 & 96.5 \\
\hline 22 & 190 & 6 & 0.07 & 30 & 100 & 97.4 & 97.4 \\
\hline 23 & 190 & 6 & 0.07 & 35 & 100 & 97.4 & 97.4 \\
\hline
\end{tabular}

TABLE 4: Reuse of NaY zeolite catalyst.

\begin{tabular}{lcc}
\hline Run & MDA conversion (\%) & MBDMA yield (\%) \\
\hline 1 & 100 & 97 \\
2 & 100 & 94 \\
3 & 100 & 90 \\
\hline
\end{tabular}

Reaction Conditions. DMC $27.0 \mathrm{~g}(0.30 \mathrm{~mol}), \mathrm{MDA} 1.98 \mathrm{~g}(0.01 \mathrm{~mol}), \mathrm{NaY}$ $1.98 \mathrm{~g}$, reaction time $6 \mathrm{~h}$, and reaction temperature $190^{\circ} \mathrm{C} . m(\mathrm{NaY}) / m(\mathrm{DMC})$ $(\mathrm{g} / \mathrm{g})=0.07 / 1$

in the room temperature, and employed in the next run. The $\mathrm{NaY}$ catalyst was reused for two more times and the results are listed in Table 4. As it is shown, the reused NaY catalysts gave excellent MDA conversion of $100 \%$ and MBDMA yield higher than $90 \%$. However, MBDMA yield gradually decreased from $97 \%$ to $90 \%$ with the increase of the used times of $\mathrm{NaY}$. To figure out the reason for such decrease, the reused $\mathrm{NaY}$ catalysts were characterized by XRD, IR, $\mathrm{NH}_{3}-\mathrm{TPD}, \mathrm{N}_{2}$ adsorption, and elemental analysis.

Figure 5 shows the XRD patterns of the fresh and reused $\mathrm{NaY}$ catalysts. All the samples similarly showed typical diffraction peaks of Y zeolite [25], indicating that the framework structures of the reused catalysts were essentially retained.

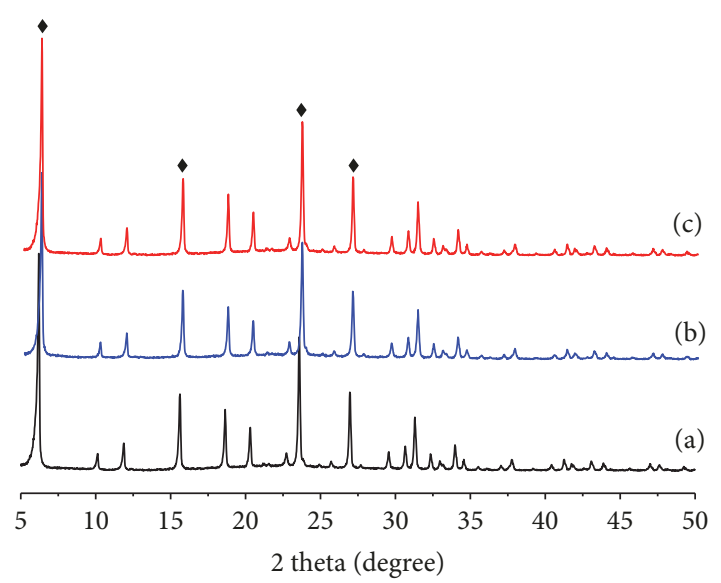

- $\mathrm{NaY}$

FIGURE 5: XRD spectra of fresh and reused NaY catalysts. (a) Fresh $\mathrm{NaY}$; (b) NaY after used for the first time; (c) NaY after used for the second time.

Figure 6 displays the IR spectra. Clearly, all the samples exhibited similar peaks at the same wave numbers of about $470 \mathrm{~cm}^{-1}, 1030 \mathrm{~cm}^{-1}, 1100 \mathrm{~cm}^{-1}, 1650 \mathrm{~cm}^{-1}$, and $3450 \mathrm{~cm}^{-1}$. The wave numbers of $470 \mathrm{~cm}^{-1}, 1030 \mathrm{~cm}^{-1}, 1100 \mathrm{~cm}^{-1}$, and 


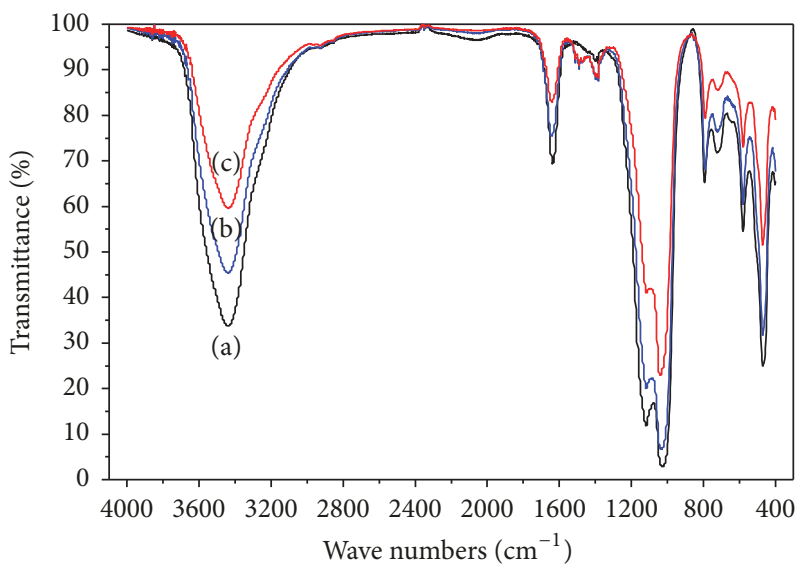

FIGURE 6: IR spectra of fresh and reused NaY catalysts. (a) Fresh $\mathrm{NaY}$; (b) NaY after used for the first time; (c) NaY after used for the second time.

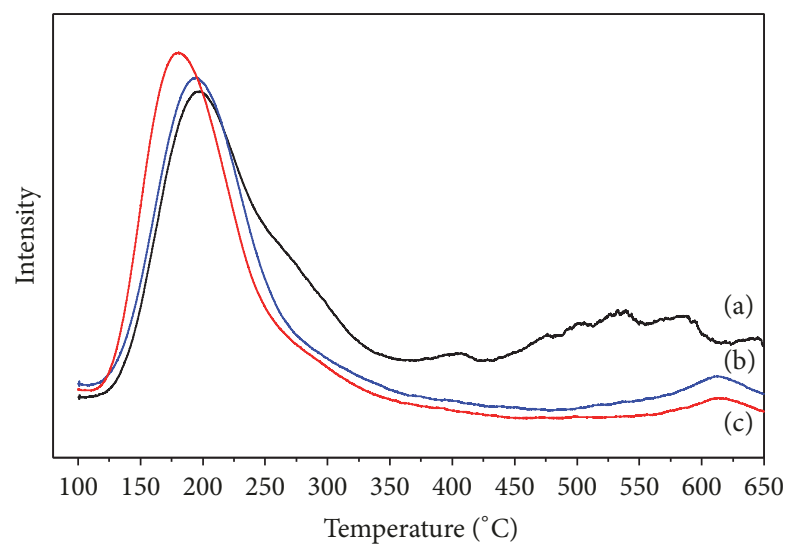

FIGURE 7: $\mathrm{NH}_{3}$-TPD profiles of fresh and reused NaY catalysts. (a) Fresh NaY; (b) NaY after used for the first time; (c) NaY after used for the second time.

$1650 \mathrm{~cm}^{-1}$ were assigned to the framework vibrations of $\mathrm{Y}$ zeolite $[25,26]$ and the $3450 \mathrm{~cm}^{-1}$ was assigned to the vibrations of the surface hydroxyl groups on $\mathrm{Y}$ zeolite [26]. The presence of those similar peaks further proved that the framework structures of the reused catalysts were essentially retained. However, the intensity of these peaks gradually attenuated with the reuse times of the $\mathrm{NaY}$ catalysts increasing, which was consistent with the decreasing trend of the MBDMA yield. The attenuation of the intensity of these IR peaks might be caused by the deposition of the compounds on the catalyst from the reaction mixture (see Table 5).

Figure 7 presents the $\mathrm{NH}_{3}$-TPD profiles. Generally, the desorption of $\mathrm{NH}_{3}$ at low temperature $\left(<200^{\circ} \mathrm{C}\right)$ was related to weak acid sites, while those at medium $\left(200^{\circ} \mathrm{C}-400^{\circ} \mathrm{C}\right)$ and high temperature $\left(>400^{\circ} \mathrm{C}\right)$ were correlated with moderate and strong acid sites. The fresh $\mathrm{NaY}$ exhibited a peak of weak acid around $190^{\circ} \mathrm{C}$, while the according peaks of reused $\mathrm{NaY}$ catalysts shifted to the low temperatures; in particular, the peak shift of the $\mathrm{NaY}$ catalysts reused for three times was rather obvious. Such shifts revealed that the acid
TABLE 5: Elemental analysis and $\mathrm{N}_{2}$ adsorption of fresh and reused $\mathrm{NaY}$ catalysts.

\begin{tabular}{lcccc}
\hline Run & C/wt\% & N/wt\% & $\begin{array}{c}\text { Surface area } \\
/ \mathrm{m}^{2} / \mathrm{g}\end{array}$ & $\begin{array}{c}\text { Pore volume } \\
/ \mathrm{cm}^{3} / \mathrm{g}\end{array}$ \\
\hline 1 & 0.1 & - & 408.2 & 0.37 \\
2 & 10.0 & 1.2 & 87.9 & 0.16 \\
3 & 10.3 & 1.3 & 54.0 & 0.15 \\
\hline
\end{tabular}

strength of reused $\mathrm{NaY}$ was gradually weakened with the used times increasing. Apparently, this weakening trend of the acid strength of the $\mathrm{NaY}$ catalysts was consistent with the decreasing trend of the MBDMA yield. Thus, it is reasonable to infer that the weakening acid strength of the $\mathrm{NaY}$ was a factor leading to the decreasing of the MBDMA yield.

The elemental analysis results of fresh and reused $\mathrm{NaY}$ catalysts are listed in Table 5. The content of the $\mathrm{C}$ in reused $\mathrm{NaY}$ catalysts sharply increased compared to that in the fresh catalysts, demonstrating the deposition of the compounds on the catalyst from the reaction mixture occurred. However, these depositing compounds cannot be detected by XRD and IR. Apparently, the deposition of the compounds would occupy some surfaces and pores, causing the decrease of the surface area and pore volume (Table 5). Accordingly, some effective active sites would be covered and the reaction place would somewhat shrink, consequently resulting in the decreasing of the MBDMA yield.

Due to a comprehensive understanding of the results of $\mathrm{XRD}, \mathrm{IR}, \mathrm{NH}_{3}-\mathrm{TPD}, \mathrm{N}_{2}$ adsorption, and elemental analysis, a full conversion of MDA was guaranteed by the essential retaining of the framework structures of the reused catalysts. The decrease of the MBDMA yield could be attributed to two factors: (1) the acid strength of reused $\mathrm{NaY}$ gradually weakened and (2) the deposition of the compounds from the reaction mixture covered some effective active sites and somewhat shrank the reaction space.

3.5. The Mechanism of N-Methylation Reaction of MDA with $D M C$. The $\mathrm{N}$-methoxycarbonylation and the $\mathrm{N}$-methylation were two competitive processes in the reaction of MDA with DMC. As described above, the full N-methylation of MDA with DMC occurred predominantly under the catalysis of $\mathrm{NaY}$, while the $\mathrm{N}$-methoxycarbonylation process was greatly restrained, obtaining a yield of the MBDMA as high as $97 \%$. Such result presented a sharp contrast with the reaction of MDA with DMC catalyzed by zinc acetate [1-3], in which the $\mathrm{N}$-methoxycarbonylation was the predominant process. Apparently, catalysts played a key role in the reaction of MDA with DMC. The zinc acetate effectively activated the carbonyl group of DMC and thus N-methoxycarbonylation product was easily formed by the direct attack of amino group of MDA on the carbonyl carbon of DMC [3,27].

However, the reaction of MDA with DMC on $\mathrm{NaY}$ was more complicated than that on zinc acetate. $\mathrm{NaY}$ can activate the molecule of DMC [20], which basically endowed it with enough activity to catalyze the reaction of MDA with DMC. However, this cannot guarantee a directional selectivity to a 


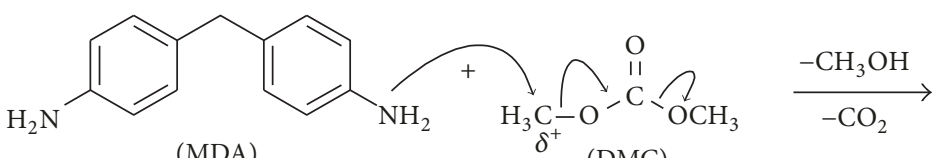

(MDA)

(DMC)<smiles>CNc1ccc(Cc2ccc(N)cc2)cc1</smiles><smiles>CN(C)c1ccc(Cc2ccc(N(C)C)cc2)cc1</smiles>

Pathway (i)

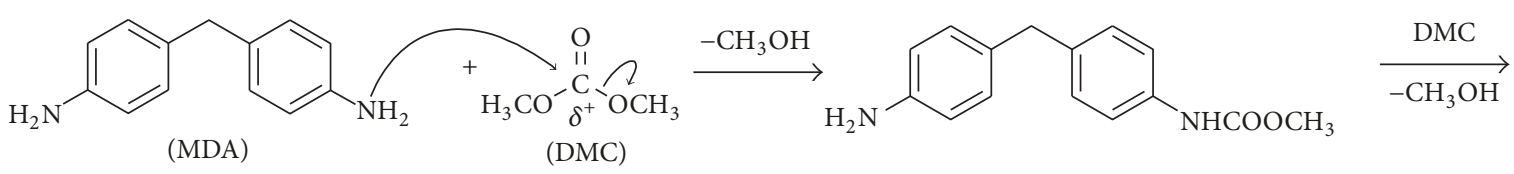<smiles>CCOC(=O)Nc1ccc(Cc2ccc(NC(=O)OC)cc2)cc1</smiles><smiles>CNc1ccc(Cc2ccc(NC(C)C(=O)O)cc2)cc1</smiles>

SCHEME 2: Two possible pathways of the N-methylation process of MDA with DMC in the presence of NaY.

particular product, which was closely related to its particular pore structures.

Generally, the $\mathrm{N}$-methylation reactions of aromatic amines with DMC may follow two possible pathways [16, 18, 27]: (i) the amino group of aromatic amine directly attacks the methoxy group of DMC. (ii) The amino group of aromatic amine firstly attacks the carbonyl group of DMC, producing $\mathrm{N}$-methoxycarbonylation intermediate. And then, the amino group of this intermediate further attacks the methyl of DMC, obtaining highly selective mono-N-methylated products. It should be noted that the selective mono-N-methylation reaction occurs in the octahedral zeolite cages of $\mathrm{NaY}$ in pathway (ii) $[16,18]$, making the particular pore structures of $\mathrm{NaY}$ to be a decisive factor in the selective synthesis of mono$\mathrm{N}$-methylated derivatives.

As mentioned above, N-methylation process of MDA with DMC occurred gradually over the NaY catalyst, successively forming partially $\mathrm{N}$-methylated products $\mathbf{1}, \mathbf{2}$, and 3 before the final product MBDMA was formed. In contrast, the peak of MDC was not detected by HPLC. MDC was the $\mathrm{N}$-methoxycarbonylated intermediate which had to be formed to selectively produce mono-N-methylated product of compound 1 (MDMA), according to the pathway (ii) (shown in Scheme 2). Such results revealed that the Nmethylation process of MDA with DMC obeyed the pathway (i), which is presented in Scheme 2. In this pathway (i), a high selectivity to the mono-N-methylated product of MDMA could not be achieved.

\section{Conclusion}

In this work, an access to $4,4^{\prime}$-methylene bis(N,N-dimethylaniline) (MBDMA) via N,N-methylation of $4,4^{\prime}$-methylenedianiline (MDA) with dimethyl carbonate (DMC) over $\mathrm{NaY}$ catalyst was reported. This process features a high yield of the required product under mild reaction conditions and sets an example of the N,N-methylation reaction of diamine with DMC. The reusability of the catalyst and the simple operations are also the advantages of this method. Also, a reasonable reaction pathway was proposed based on the 
identification of the by-products and the tracing analysis of the transformation of the by-products during the reaction process. Furthermore, the implementation of such process proved that the selectivity to the two competitive processes $\mathrm{N}$ methylation and $\mathrm{N}$-methoxycarbonylation can be controlled by changing the catalysis systems and the reaction conditions.

\section{Conflicts of Interest}

The authors declare that there are no conflicts of interest regarding the publication of this paper.

\section{Acknowledgments}

This work is supported by the Chinese National Natural Science Foundation (no. 51403229 and no. 21606177) and the Scientific Research Training Program of Xian University of Petroleum.

\section{References}

[1] I. Vauthey, F. Valot, C. Gozzi, F. Fache, and M. Lemaire, "An environmentally benign access to carbamates and ureas," Tetrahedron Letters, vol. 41, no. 33, pp. 6347-6350, 2000.

[2] T. Baba, A. Kobayashi, T. Yamauchi et al., "Catalytic methoxycarbonylation of aromatic diamines with dimethyl carbonate to their dicarbamates using zinc acetate," Catalysis Letters, vol. 82, no. 3-4, pp. 193-197, 2002.

[3] Z. Qiu, J. Wang, M. Kang, Q. Li, and X. Wang, "Formation of intermediate and by-products in synthesis of 4,4/methylenedimethyldiphenylcarbamate," Catalysis Letters, vol. 124, no. 3-4, pp. 243-249, 2008.

[4] W. Buchberger, "Determination of iodide and bromide by ion chromatography with post-column reaction detection," Journal of Chromatography A, vol. 439, no. 1, pp. 129-135, 1988.

[5] L. Su, J. Li, H. Ma, and G. Tao, "Determination of trace amounts of manganese in natural waters by flow injection stopped-flow catalytic kinetic spectrophotometry," Analytica Chimica Acta, vol. 522, no. 2, pp. 281-288, 2004.

[6] R. Periasamy, S. Kothainayaki, and K. Sivakumar, "Investigation on inter molecular complexation between 4,4/-methylenebis(N,N-dimethylaniline) and $\beta$-cyclodextrin: Preparation and characterization in aqueous medium and solid state," Journal of Molecular Structure, vol. 1080, pp. 69-79, 2014.

[7] S. D. Choudhury and S. Basu, "Caging of phenazine by 4,4/bis(dimethylamino)diphenylmethane: A comparative study with phenazine-N,N-dimethylaniline," Chemical Physics Letters, vol. 383, no. 5-6, pp. 533-536, 2004.

[8] S. D. Choudhury and S. Basu, "Exploring the extent of magnetic field effect on intermolecular photoinduced electron transfer in different organized assemblies," The Journal of Physical Chemistry A, vol. 109, no. 36, pp. 8113-8120, 2005.

[9] D. Dey, A. Bose, M. Chakraborty, and S. Basu, "Magnetic field effect on photoinduced electron transfer between dibenzo[a,c]phenazine and different amines in acetonitrile water mixture," The Journal of Physical Chemistry A, vol. 111, no. 5, pp. 878-884, 2007.

[10] W. L. Borkowski and E. C. Wagner, "Methylation of aromatic amines by the wallach method," The Journal of Organic Chemistry, vol. 17, no. 8, pp. 1128-1140, 1952.
[11] Y. Wang, "An experimental and theoretical study on the preparation of 4,4/-methylene-bis(N,N-dimethylaniline) in ionic liquid," Journal of Physical Organic Chemistry, vol. 29, p. p. 276, 2016.

[12] H. Badawi, "A comparative study of the structure and vibrational spectra of diphenylmethane, the carcinogen 4,4/methylenedianiline and 4,4/-methylene-bis(N,N-dimethylaniline)," Spectrochimica Acta Part A: Molecular and Biomolecular Spectroscopy, vol. 109, p. 213, 2013.

[13] P. Tundo and M. Selva, "The chemistry of dimethyl carbonate," Accounts of Chemical Research, vol. 35, no. 9, pp. 706-716, 2002.

[14] K. Sreekumar, T. M. Jyothi, T. Mathew, M. B. Talawar, S. Sugunan, and B. S. Rao, "Selective N-methylation of aniline with dimethyl carbonate over $\mathrm{Zn}(1-\mathrm{x}) \mathrm{Co}(\mathrm{x}) \mathrm{Fe} 2 \mathrm{O} 4(\mathrm{x}=0,0.2$, 0.5, 0.8 and 1.0) type systems," Journal of Molecular Catalysis A: Chemical, vol. 159, no. 2, pp. 327-334, 2000.

[15] N. Nagaraju and G. Kuriakose, "Activity of amorphous V-AlPO4 and Co-AlPO4 in the selective synthesis of N-monoalkylated aniline via alkylation of aniline with methanol or dimethyl carbonate," New Journal of Chemistry, vol. 27, no. 4, pp. 765-768, 2003.

[16] M. Selva, A. Bomben, P. Tundo, and J. Chem, "Selective mono-N-methylation of primary aromatic amines by dimethyl carbonate over faujasite X- and Y-type zeolites," Journal of the Chemical Society, Perkin Transactions 1, no. 7, p. 1041, 1997.

[17] M. Selva, P. Tundo, and A. Perosa, "Reaction of primary aromatic amines with alkyl carbonates over $\mathrm{NaY}$ faujasite: $\mathrm{A}$ convenient and selective access to mono- $\mathrm{N}$-alkyl anilines," The Journal of Organic Chemistry, vol. 66, no. 3, pp. 677-680, 2001.

[18] M. Selva, P. Tundo, and A. Perosa, "Mono-N-methylation of primary amines with alkyl methyl carbonates over $\mathrm{Y}$ faujasites. 2. Kinetics and selectivity," The Journal of Organic Chemistry, vol. 67, no. 26, pp. 9238-9247, 2002.

[19] M. Selva, P. Tundo, and A. Perosa, "Reaction of Functionalized Anilines with Dimethyl Carbonate over NaY Faujasite. 3. Chemoselectivity toward Mono- $N$-methylation," Journal of Organic Chemistry, vol. 68, no. 19, p. 7374, 2003.

[20] M. Selva, P. Tundo, and T. Foccardi, "Mono-N-methylation of functionalized anilines with alkyl methyl carbonates over $\mathrm{NaY}$ faujasites. 4. Kinetics and selectivity," The Journal of Organic Chemistry, vol. 70, no. 7, pp. 2476-2485, 2005.

[21] A. J. Cabrero, R. Adam, K. Junge, and M. Beller, "A general protocol for the reductive $\mathrm{N}$-methylation of amines using dimethyl carbonate and molecular hydrogen: mechanistic insights and kinetic studies," Catalysis Science and Technology, vol. 6, no. 22, p. 7956, 2016.

[22] I. I. Ivanova, E. B. Pomakhina, A. I. Rebrov, W. Wang, M. Hunger, and J. Weitkamp, "Mechanism of Aniline Methylation on Zeolite Catalysts Investigated by In Situ 13C NMR Spectroscopy," Kinetics and Catalysis, vol. 44, no. 5, pp. 701-709, 2003.

[23] T. Esakkidurai and K. Pitchumani, "Zeolite-promoted selective mono-N-methylation of aniline with dimethyl carbonate," Journal of Molecular Catalysis A: Chemical, vol. 218, no. 2, pp. 197201, 2004.

[24] O. A. Ponomareva, P. A. Shaposhnik, M. V. Belova1, B. A. Kolozhvari, and I. I. Ivanova, "Novel method for the preparation of Cs-containing FAU(Y) catalysts for aniline methylation," Frontiers of Chemical Science and Engineering, vol. 12, no. 1, pp. 70-76, 2018, https://link.springer.com/article/10.1007\% 2Fs11705-017-1694-3. 
[25] D. Guo, B. Shen, Y. Qin et al., "USY zeolites with tunable mesoporosity designed by controlling framework Fe content and their catalytic cracking properties," Microporous and Mesoporous Materials, vol. 211, pp. 192-199, 2015.

[26] R. Xu and W. Pang, Chemistry-zeolites and porous materials, Science press, Beijing, China, 2004.

[27] T. Baba, A. Kobayashi, Y. Kawanami et al., "Characteristics of methoxycarbonylation of aromatic diamine with dimethyl carbonate to dicarbamate using a zinc acetate catalyst," Green Chemistry, vol. 7, no. 3, pp. 159-165, 2005. 

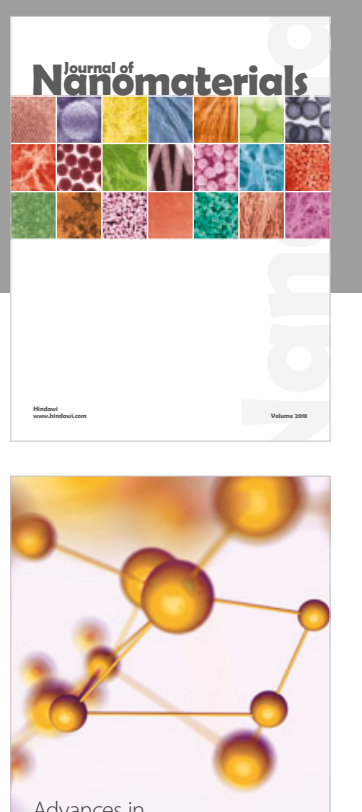

Physical Chemistry
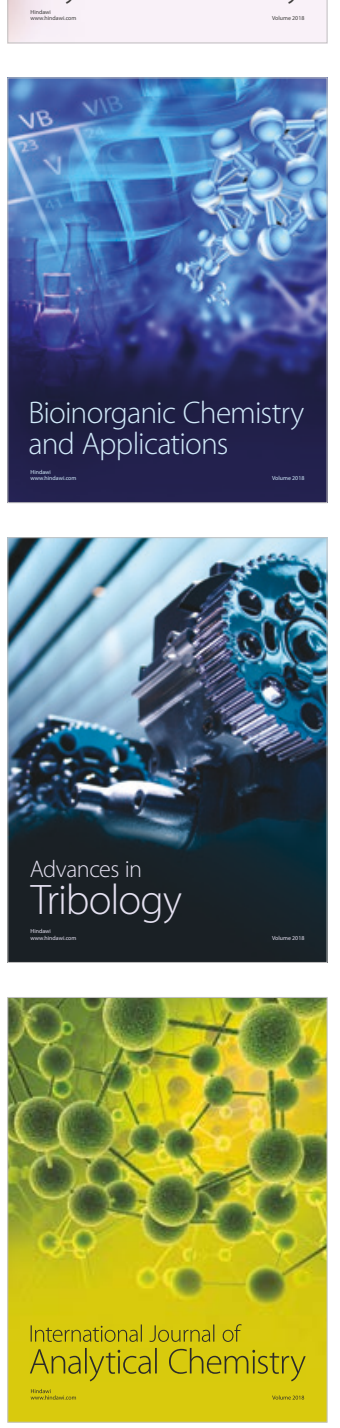

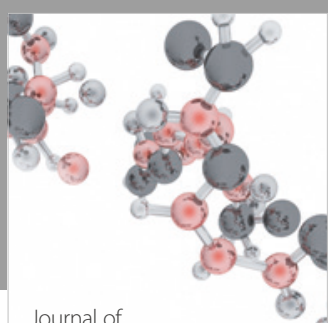

Analytical Methods

in Chemistry

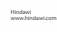

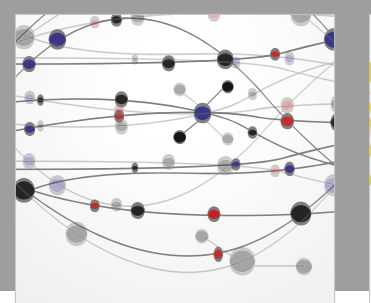

The Scientific World Journal

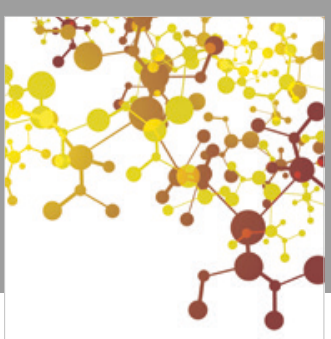

Journal of

Applied Chemistry
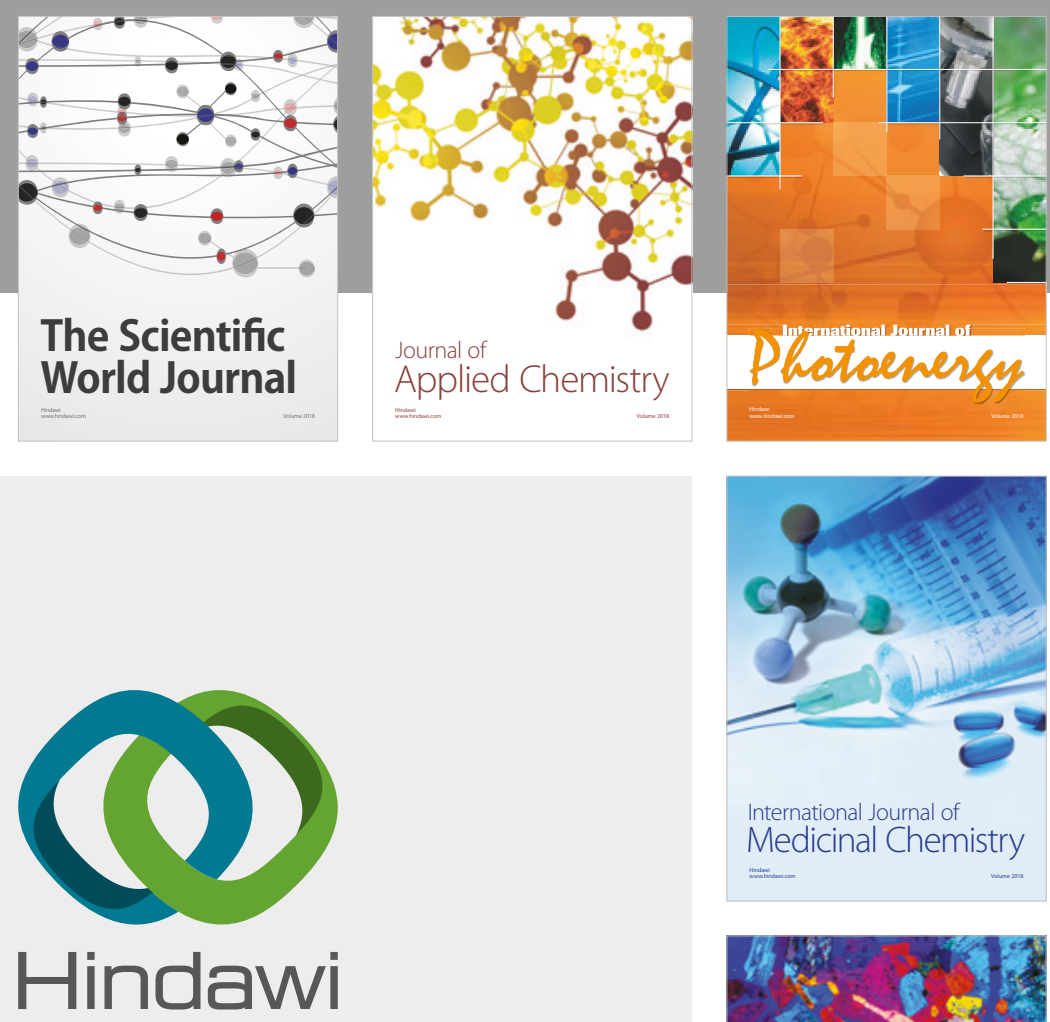

Submit your manuscripts at

www.hindawi.com
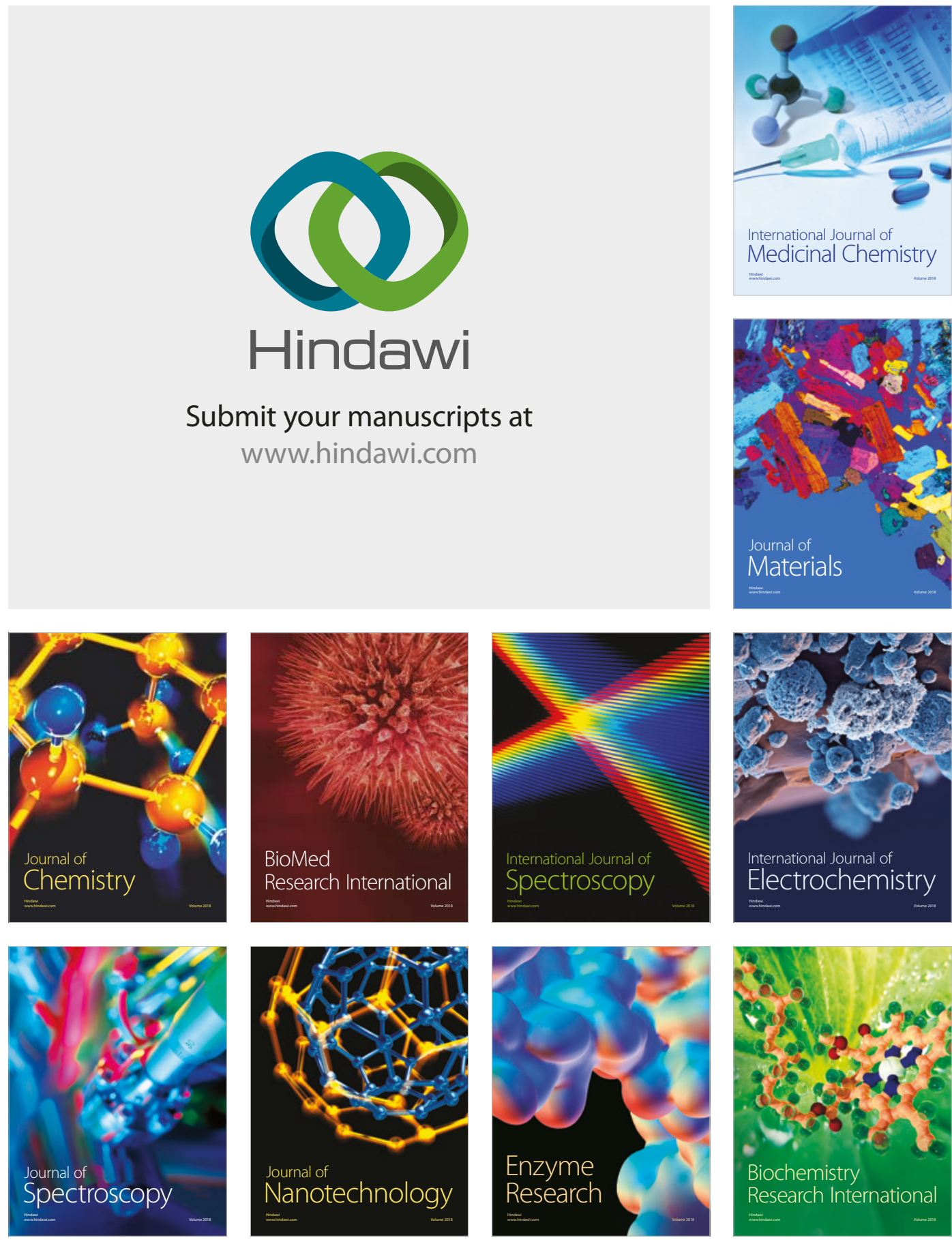
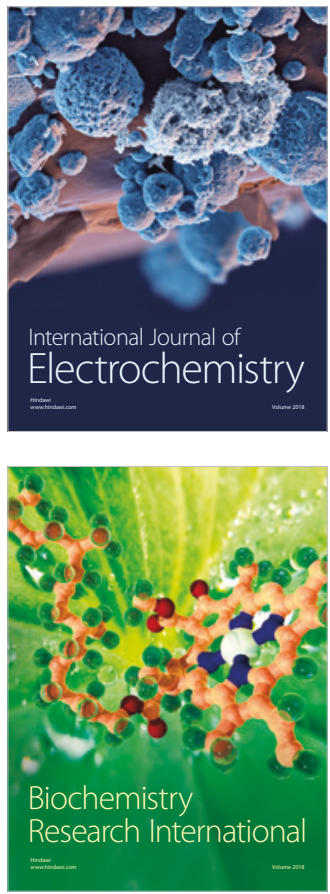